\title{
Trastornos relacionados con sustancias y trastornos adictivos
}

\author{
Substance-related and addictive disorders
}

\section{Elisardo Becoña}

\section{DEFINICIÓN Y CAMBIOS EN RELACIÓN AL DSM-IV}

Como ocurre con la publicación de cada nuevo DSM, un poco antes de su publicación y poco después de la misma, hay una importante discusión sobre sus ventajas, desventajas, utilidad, problemas, etc. Esto es lo que está ocurriendo con la reciente publicación del DSM-5, aunque esta vez las críticas al mismo están siendo de mayor calado.

En la denominación de trastornos relacionados con sustancias y trastornos adictivos se incluyen las distintas sustancias psicoactivas y el juego (antes juego patológico, que aparecía en el apartado de trastornos de control de impulsos no clasificados en otros apartados, en el DSM-IV). Aquí solo nos centraremos en los trastornos relacionados con sustancias (TRS) por cuestiones de espacio.

Cualquier cambio en los criterios diagnósticos del TRS es relevante, por ser un trastorno muy prevalente y si hay cambios pueden alterarse los datos de prevalencia; su diagnóstico tiene no solo implicaciones clínicas sino también legales (ej., para la aplicación de eximentes); y, cara al tratamiento, los cambios pueden significar que más personas tengan que ir a tratamiento (ej., en fu-

\section{Catedrático de Psicología Clínica}

Unidad de Tabaquismo y Trastornos Adictivos

Facultad de Psicología, Universidad de Santiago de Compostela

Corres pondencia: Dr. D. Elisardo Becoña

Unidad de Tabaquismo y Trastornos Adictivos

Facultad de Psicología. Campus Vida

Calle Xosé María Suárez Núñez, s/n

15782 Santiago de Compostela

Email: elisardo.becona@usc.es madores de tabaco, bebedores de alcohol, consumidores de cannabis, etc.).

Los principales cambios que el DSM-5 introduce son los siguientes (American Psychiatric Association, 2000, 2013; Compton, Sawson, Goldstein y Grant, 2013; Hasin et al., 2013):

1) Se elimina el abuso y dependencia del DSMIV y solo queda el TRS como una suma de criterios de abuso y dependencia del DSM-IV. El diagnóstico se hace cuando la persona tiene 2 o más síntomas de 11 a lo largo de un periodo de tiempo de 12 meses.

2) Aparece el nivel de severidad: bajo (2-3), moderado (4-5) o severo (6 o más) en función del número de criterios diagnósticos. Se ha sugerido que la categoría de baja equivale al abuso del DSMIV, y las de moderado y severo a las de dependencia del DSM-IV.

3) Los 11 criterios proceden de unir los de abuso y dependencia del DSM-IV con las siguientes excepciones: a) se elimina el ítem del DSM-IV referido a tener problemas legales (aduciéndose que apenas se utilizaba), y b) se añade el ítem de craving.

4) Los TRS se dividen en dos grupos: a) trastornos por consumo de sustancias, $\mathrm{y} b$ ) trastornos inducidos por sustancias (intoxicación, abstinencia, otros trastornos mentales inducidos por una sustancia o medicación). Se mantienen la mayoría de los síndromes psiquiátricos inducidos por sustancias del DSM-IV (ej., depresión inducida por consumo de sustancias) y se amplían a otros (ej., trastorno bipolar inducido por el consumo de sustancias).

5) Aparece por primera vez en el DSM-5 el trastorno de juego dentro de la categoría de trastorno por consumo de sustancias. 
6) Las sustancias pasan de 11 en el DSM-IV a 10 en el DSM-5, al hacer una reordenación de las mismas. Quedan las siguientes: alcohol; cafeína; cannabis; alucinógenos (fenciclidina y otros alucinógenos); inhalantes; opiáceos; sedantes; hipnóticos y ansiolíticos; estimulantes (sustancia anfetamínica, cocaína y otros estimulantes), tabaco y otras sustancias (o sustancias desconocidas).

7) Se introduce el síndrome de abstinencia del cannabis (que no aparecía en el DSM-IV). El síndrome de abstinencia para la cafeína no se incluye, solo aparece en el apartado $\mathrm{C}$ del manual.

En la tabla 1 puede verse un cuadro comparativo entre el DSM-IV y el DSM-5.

\section{IMPLICACIONES DE LOS CAMBIOS INTRODUCIDOS}

Son varias las implicaciones que tiene para la clínica y para los estudios de prevalencia los nue- vos criterios diagnósticos, junto con algunos problemas que surgen con los mismos.

El punto de vista jerárquico de abuso y dependencia era considerado problemático (Jones, Gill y Ray, 2012), de ahí que se haya eliminado. Pero, aunque la mayoría de los clínicos asumían que un diagnóstico de abuso era un precursor del posterior diagnóstico de dependencia, los estudios no dicen esto. Por ejemplo, en el de Schuckit et al. (2001) solo el $10 \%$ de las personas diagnosticadas de abuso del alcohol se convertían posteriormente (3-5 años de seguimiento) en personas dependientes del alcohol. La justificación de la unidimensionalidad para el TRS se basó en distintos análisis factoriales y en los estudios con la teoría de la respuesta al ítem. En ambos aparecía un solo factor que incluye a ambas categorías (Hasin et al., 2013).

Otro importante punto de discusión es el punto de corte. Ahora son 2 de 11 criterios. Los estudios de validación realizados, utilizando tanto mues-

Tabla 1

Criterios del DSM-IV y DSM-5 para el trastorno por consumo de sustancias

\begin{tabular}{|c|c|c|c|}
\hline & $\begin{array}{c}\text { DSM-IV } \\
\text { Abuso }^{\mathrm{a}} \\
(\geq 1 \text { criterio })\end{array}$ & $\begin{array}{c}\text { DSM-IV } \\
\text { Dependencia }^{\mathrm{b}} \\
(\geq 3 \text { criterios })\end{array}$ & $\begin{array}{c}\text { DSM-5 } \\
\text { Trastorno por } \\
\text { consumo de } \\
\text { sustancias }^{\mathrm{c}} \\
(\geq 2 \text { criterios })\end{array}$ \\
\hline Uso peligroso & $\mathrm{X}$ & & $\mathrm{X}$ \\
\hline Problemas sociales/interpersonales relacionados con el consumo & $\mathrm{X}$ & & $\mathrm{X}$ \\
\hline Incumplimiento de los principales roles por su consumo & $\mathrm{X}$ & & $\mathrm{X}$ \\
\hline Problemas legales & $\mathrm{X}$ & & \\
\hline Síndrome de abstinencia ${ }^{\mathrm{d}}$ & & $\mathrm{X}$ & $\mathrm{X}$ \\
\hline Tolerancia & & $\mathrm{X}$ & $\mathrm{X}$ \\
\hline Usa mayor cantidad/más tiempo & & $\mathrm{X}$ & $\mathrm{X}$ \\
\hline Intentos repetidos de dejarlo/controlar el consumo & & $\mathrm{X}$ & $\mathrm{X}$ \\
\hline Mucho más tiempo del que pensaba & & $\mathrm{X}$ & $\mathrm{X}$ \\
\hline Problemas físicos/psicológicos relacionados con el consumo & & $\mathrm{X}$ & $\mathrm{X}$ \\
\hline Deja de hacer otras actividades debido al consumo & & $\mathrm{X}$ & \\
\hline Craving & & & $\mathrm{X}$ \\
\hline
\end{tabular}

${ }^{a}$ Uno o más criterios de abuso dentro de un periodo de 12 meses y sin diagnóstico de dependencia. Aplicable a todas las sustancias excepto a la nicotina.

${ }^{\text {b}}$ Tres o más criterios de dependencia dentro de un periodo de 12 meses.

${ }^{\mathrm{c}}$ Dos o más criterios del trastorno por consumo de sustancias en un periodo de 12 meses.

${ }^{\mathrm{d}}$ El síndrome de abstinencia no está incluido para el cannabis, inhalantes y trastornos alucinógenos del DSMIV. El síndrome de abstinencia del cannabis se ha añadido en el DSM-5.

Adaptado de Hasin et al. (2013, p. 836). 
tras de la población general como clínicas, cambiaba poco la prevalencia de usar 2, 3 o 4 criterios como punto de corte para el TRS (Hasin et al., 2013). Pero otros estudios, con muestras muy amplias (ej., Kerridge, Saha, Gmel y Rehm, 2013), indican para el alcohol que el punto adecuado con criterios DSM-5 sería el de 4, no 2. Y, en estudio de Compton et al. (2013), también con una amplia muestra, con criterios DSM-5, el punto de corte sería de 4 o más para el alcohol, cocaína y opiáceos. Para el cannabis sería de 6 o más criterios. Lo que pretende el DSM-5 al bajar el punto de corte es apresar a los "diagnósticos huérfanos" (Agrawal, Health y Lynskey, 2011). El problema ahora está en que hay el riesgo de diagnosticar esos diagnósticos huérfanos y otros adicionales que no tengan esas personas.

La limitación anterior intenta solventarse con la introducción del nivel de severidad del trastorno: leve (2-3), moderado (4-5) y severo (6 o más criterios). Claramente, esto lleva a que el tratamiento no puede ser el mismo para los distintos niveles de gravedad. Se añade confusión y llevará a que, utilizar 2 de 11 criterios, no sea correcto en todos los casos para el diagnóstico, incrementándose artificialmente el porcentaje de personas con el trastorno. Esto va a ocurrir sobre todo en personas jóvenes. Además, estos criterios olvidan las características del inicio en el consumo de cualquier sustancia (donde la intoxicación, la tolerancia y el síndrome de abstinencia son característicos), sobre todo en jóvenes; y a que el DSM-5 se ha olvidado de la relevancia de las variables sociales, contextuales y psicológicas que se relacionan con el inicio del consumo de sustancias (Becoña, 2002). Por ello, en el caso del alcohol, y del tabaco (donde antes en este no había el diagnóstico de abuso), como en otras drogas (ej., cannabis), la prevalencia con el DSM-5 va a incrementarse de modo significativo. Esto llevará a la estigmatización de personas "normales" o a, simplemente, no aceptar el diagnóstico en los casos límites o dudosos.

En una línea semejante está la introducción del craving como criterio diagnóstico. Lo que dicen los miembros de grupo de elaboración del TUS para el DSM-5 (Hasin et al., 2013) es bien elocuente: "los clínicos expresaron su entusiasmo en añadir el craving en las presentaciones de trabajo y en la web del DSM-5. Finalmente, a pesar de que los beneficios psicométricos de añadir el criterio de craving es ambiguo, la consideración de que el craving puede ser un objetivo del tratamiento biológico (una perspectiva no psicométrica) prevaleció. Mientras se espera que se produzca el desarrollo de indicadores de craving biológicos, los clínicos e investigadores pueden evaluar el craving con cuestionarios, como los utilizados en los estudios de la teoría de respuesta al ítem" (pp. 840). Sobran comentarios.

También se abre un nuevo frente a nivel legal $\mathrm{y}$ forense, como ya hemos comentado previamente.

Con el DSM-5 se ha perdido una buena oportunidad de hacer tipologías de adictos, que son más útiles en la clínica, y que integran la tan habitual comorbilidad asociada. Estudios como el de Moss, Chen y Yi (2007), en la dependencia del alcohol, como se ha hecho en otras sustancias, indica que es posible hacerlo, como ya se ha hecho en otros trastornos (ej., en los tipos de esquizofrenia, tipos de trastorno bipolar, etc., del propio DSM-5).

Como ya indicamos, va a ser difícil en la práctica distinguir si la persona tiene TRS por un consumo "médico" de un fármaco psicoactivo recetado, o si lo tiene porque se lo toma "automedicado por su cuenta" o si es un adicto realmente (ej., en la morfina). Es uno de los puntos más débiles para el diagnóstico y más confuso al usar dos varas de medir para lo mismo. ¿Por qué en unos casos hacer el diagnóstico y en otros no?, ¿dónde está la fiabilidad del diagnóstico?

No extraña por ello que el DSM-5 reciba críticas, y que se vea que el DSM-10 es más útil o que lo puede ser el próximo DSM-11. El tiempo lo dirá.

En conclusión, estamos ante nuevos criterios diagnósticos para el TRS. Su utilidad la veremos en los próximos años, así como su grado de aceptación. Los problemas que hoy se plantean son semejantes a los que aparecen cuando hay nuevos criterios DSM. Categorizar la realidad clínica, la de los trastornos mentales y del comportamiento, no es tarea fácil. Más difícil aún lo es en un trastorno con tan alta carga social (ej., disponibilidad y precio de la sustancia, alto nivel de prevalencia) y psicológica (ej., expectativas, percepción de riesgo de cada sustancia, procesos de aprendizaje). Sin negar el claro peso biológico de este trastor- 
no, una mera explicación biológica es insuficiente para explicar esta compleja conducta, y menos tal como se han propuesto los criterios del TRS, especialmente para la categoría de "ligera".

\section{BIBLIOGRAFÍA}

1. Agrawal, A., Health, A.C. y Lynskey, M.T. (2011). DSM-IV to DSM-5. Addiction, 106: 1935 1943.

2. American Psychiatric Association (2000). Diagnostic and statistical manual for mental disorders, 4th edition, Revised Text. Washington, D.F: Autor (trad. cast.: Barcelona, Masson, 2001).

3. American Psychiatric Association (2013). Diagnostic and statistical manual for mental disorders, 5th edition, DSM-5. Washington, D.F: American Psychiatric Publishing.

4. Becoña, E. (2002). Bases científicas de la prevención del consumo de drogas. Madrid: Delegación del Gobierno para el Plan Nacional sobre Drogas.

5. Compton, W.M., Dawson, D.A., Goldstein, R.B. y Grant, B.F. (2013). Crosswalk between DSM-IV dependence and DSM-5 substance use disorders for opioids, cannabis, cocaine and alcohol. Drug and Alcohol Dependence, 122: 38.46 .
6. Hasin, D.S., O’Brien, C.P. Auriacombe, M., Borges, G., Bucholz, K., Budney, A., y Grant, B.F. (2013). DSM-5 criteria for substance use disorders: Recommendations and rationale. American Journal of Psychiatry, 170: 834-851.

7. Jones, K.D., Gill, C. y Ray, S. (2012). Review of the proposed DSM-5 substance use Disorder. Journal of Addictions \& Offender Counseling, 33: 115-133.

8. Kerridge, B.T., Saha, T.D., Gmel, G. y Rehm, J. (2013). Taxometric analysis of DSM-IV and DSM-5 alcohol use disorders. Drug and Alcohol Dependence, 129: 60-69.

9. Kupfer, D.J., Kuhl, E.A. y Regier, D.A. (2013). DSM-5-the future arrived. JAMA, 309: 16911692.

10. Moss, H.B., Chen, C.M. y Yi, H-J. (2007). Subtypes of alcohol dependence in a nationally representative simple. Drug and Alcohol Dependence, 91: 149-158.

11. Sandín, B . (2013). DSM-5: ¿Cambio de paradigma en la clasificación de los trastornos mentales? Revista de Psicopatología y Psicología Clínica, 18: 255-286.

12. Schuckit, M.A., Smith, T.L., Danko, G.P., Bucholz, K.K., Reiche, T. y Bierut, L. (2001). Five-year clinical course associated with DSM-IV-TR alcohol abuse and dependence in a large group of men and women. American Journal of Psychiatry, 162: 350-360. 